\section{Oncology and haematology}

\section{G48 THE EFFECT OF CHANGING PROTOCOLS ON THE NEED FOR HIGH DEPENDENCY AND INTENSIVE CARE IN CHILDREN WITH CANCER: A REGIONAL REVIEW}

L. Newbury, P. Sartori, R.I. Ross Russell. Department of Paediatrics, Addenbrooke's Hospital. Cambridge, CB2 2QQ

Introduction: Over the last few years there has been a trend towards more intensive treatment for paediatric oncology patients. This includes both primary and relapsed disease protocols. The impact of these changes on the usage of intensive care (PIC) and high dependency care (HD) is unclear.

Aim: To quantify the changes in PIC and HD usage for oncology patients over the last 3 years.

Methods: This hospital houses the only tertiary paediatric oncology unit as well as the only PIC unit for the region. We have reviewed the admissions to our combined PIC/HD unit over the last 3 years. The reason for admission, risk of mortality, length of stay and outcome were recorded.

Results: Annual admissions of Oncology patients to the unit increased from 32 in 1999 to 72 in 2001 (2.2 fold increase). Bed days over this period increased from 61 to 302 (5 fold increase). The percentage of patients admitted for sepsis rose from $3 \%$ to $26 \%$. Mortality on PICU remains quite low at $7 \%$ (standardised mortality rate against PIM, 1.04) but a further $5 \%$ of patients die within 1 month of discharge. Median length of stay has not changed (1 day for surgical and 2 days for medical patients) however a small number of long stay patients skew the data. In 20018 patients (11\%) accounted for $59 \%$ of bed days

Conclusion: Although many oncology children requiring $\mathrm{HD}$ continue to be nursed on a non HD ward, there is a substantial rise in admissions to our PIC unit. This probably reflects the increasing complexity of protocols and the rise in treatment of relapsed disease.

\section{G49 INACCURACY OF MEASUREMENT OF GLOMERULAR FILTRATION RATE IN PATIENTS WITH LARGE SOLID TUMOURS}

M. Burniston, S. Picton, U. Reid, M. Barnfield. St James's University Hospital, Leeds, West Yorkshire

Aims: Assessment of glomerular filtration rate (GFR) to monitor the nephrotoxic effect of drugs or as a basis for drug dosing plays an important role in the management of oncology patients. Measurement of GFR by isotope tracer techniques is regarded as a gold standard but commonly occurring conditions in oncology patients may alter tracer kinetics and lead to inaccuracies of measurement. The aim of this study was to establish if clinically significant errors are made in patients with large solid tumours.

Method: Ten newly diagnosed paediatric oncology patients had pre-treatment GFR measured using radiolabelled tracers with an extended sampling technique. This served as a reference technique and was compared to the result obtained using the standard technique of sampling between two and four hours. In eight of these patients measurements were repeated after several cycles of chemotherapy treatment.

Results: In all ten patients there were significant deviations of the pattern of tracer plasma clearance from the expected single exponential after two hours. The GFR calculated from the standard technique was significantly higher (median 33\%, range 11-44\%) than the GFR calculated by the reference technique.

In all eight post-therapy patients the clearance more closely approached the expected pattern and the difference between the reference and standard GFR calculations was significantly reduced (median $22 \%$, range $-7 \%-34 \%$ ).

Conclusions: In this group of patients the tracer kinetics were disrupted from the expected pattern and this led to clinically significant errors in the standard technique of measuring GFR. This may be due to altered vascularity or changes in permeability due to the disease state. Reductions in measured GFR with treatment require careful interpretation as they may not be due to nephrotoxicity but to reduction of tumour bulk. If accurate assessments of GFR are required for monitoring nephrotoxic effect or for chemotherapy dosing, an extended sampling schedule may be required.
G50 SEROLOGICAL DEMONSTRATION OF NEONATAL ABO BLOOD GROUP ANTIBODIES IN CORD BLOOD SAMPLES

J. Skiming, B. Fung, J. Minards, P.H.B. Bolton-Maggs. Department of Haematology, Royal Liverpool Children's Hospital, Alder Hey, Liverpool and Liverpool Women's Hospital

Aims: While A and $B$ red cell antigens are determined in fetal life, it is not clear when infants develop the corresponding blood group antibodies ( $\lg M)$. Do these develop over the first few months of life in response to exogenous antigens, or is the ability innate? Generally infants are grouped on the basis of red cells alone because the antibodies are either weak or absent, but there is very little available evidence. The aim of this study was to determine what proportion of infants has such antibodies at birth, and how strong they are.

Methods: Cord blood samples, and corresponding maternal samples, were taken at birth from Rh D negative women. Red cell groups and antiglobulin testing were performed by routine standard tests. Sera were analysed for both $\lg G$ and $\lg M$ antibodies by testing for agglutination with $\mathrm{ABO}$ cell solutions containing saline with bromelain (necessary for lgG to cause agglutination-presumed maternal antibodies). Positive samples were tested in saline alone; agglutination then would be caused only by the presence of $\lg M$ antibodies of fetal origin (because maternal $\lg M$ does not cross the placenta). Samples positive in saline only were then titred, the titre being recorded as the reciprocal of the highest dilution giving weak agglutination.

Results: 195 cord samples were tested. The red cell groups showed $56 \%$ infants to be group $0,34 \%$ group $A, 8 \%$ group $B$ and $2 \%$ group $A B$. $140 / 195(72 \%)$ serum samples showed reactions in bromelain, but 91 (47\%) agglutinated cells in saline only and therefore contained fetal $\lg M$. These babies blood groups were $\mathrm{O}$ in $71(78 \%)$, group $A, 14(15 \%)$ and group $B, 6(7 \%)$. The highest titre of antibodies was 128 (anti- $A$ in a group $O$ infant), and the median titres for anti-A and anti-B were 1 and 0 . Most infants had low titre agglutinins. (The normal adult or older child of group $O$ has anti-A titres of at least 8-16 and anti-B titres of 4-8).

Conclusion: $A B O$ antibodies of fetal origin are present in nearly half of normal neonates, and although mainly in low titre, some were high. These findings support the view that the antibody production is innate.

\section{G51 BONE MINERAL DENSITY (BMD) IN CHILDHOOD SURVIVORS OF ACUTE LYMPHOBLASTIC LEUKAEMIA (ALL) TREATED WITHOUT CRANIAL IRRADIATION (XRT)}

B.M.D. Brennan, M.Z. Mughal, C. Bean, E. Auld, S.M. Shalet, O.B. Eden R.F. Stevens, A. Will, S.A. Roberts, J.E. Adams. Central Manchester \& Manchester Children's University Hospitals

Aims: We have previously shown that adult survivors of childhood ALL whose treatment included cranial XRT have a highly significant reduction in volumetric spinal BMD. As cranial XRT has been implicated as a factor for reduced BMD in ALL survivors, we examined the BMD in 56/25 males] childhood survivors of ALL who had completed their treatment.

Methods: We measured bone mineral content (BMC) and bone area $(B A)$ of $L 1$ to $L 4$ vertebrae (LS) of the subjects using the Hologic QDR-4500 dual energy x-ray absorpiometry, and the volumetric trabecular BMD at the distal radius (DRvTBMD; $\mathrm{mg} / \mathrm{cm}^{3}$ ) using the Stratec XCT 2000 peripheral quantitative computer tomography scanner. The bone mineral apparent density (BMAD; $\mathrm{g} / \mathrm{cm}^{3}$ ) of $\mathrm{LS}$ (calculated by dividing $L S B M C$ by $L S B^{1.5}$ ) and DRvTBMD in each ALL subject was compared with that of gender and age matched control, using the Wilcoxon test.

Results: There were no gender differences in the LS BMAD \& DRvTBMD among the ALL subjects. See table.

Conclusions: We found significantly reduced DRvTBMD among childhood survivor of ALL treated without XRT, compared with healthy controls. As BMD is a surrogate measure of bone strength, childhood survivor of ALL may be at increased risk of sustaining distal forearm fractures.

\section{G52 AUDIT OF SEMEN CRYOPRESERVATION IN YOUNG MALES UNDERGOING GONADOTOXIC THERAPY; EXPERIENCE OF SUCCESS AND PROCESS OF OBTAINING LEGAL CONSENT}

M.D. Zalkin, G. Bahadur, H.A. Spoudeas. Department of Adolescent Endocrinology \& Oncology and Reproductive Laboratories, Middlesex Hospital, London

Background: The increased survival rates of childhood cancer and the recent advances in assisted reproductive technologies mean that 


\begin{tabular}{|llll|}
\hline \multicolumn{2}{|l|}{ Abstract G51 } & Control & $\begin{array}{l}\text { P }(95 \% \text { Cl for median } \\
\text { difference })\end{array}$ \\
\hline ALL & $11.1(8.9$ to 13.4$)$ & $11.0(9.0$ to 13.6$)$ & \\
Age & $145(33$ to 158$)$ & $146(136$ to 158$)$ & $0.57(-3.1$ to 1.7$)$ \\
Height & $42.9(32.6$ to 53.5$)$ & $39.6(31.5$ to 54.4$)$ & $0.83(-2.2$ to 5.3$)$ \\
Weight & $19.5(17.8$ to 22.1$)$ & $18.62(16.3$ to 21.0$)$ & $0.061(-0.06$ to 2.5$)$ \\
BMl & $0.21(0.19$ to 0.23$)$ & $0.21(0.20$ to 0.23$)$ & $0.14(-0.02$ to 0.003$)$ \\
LS BMAD & $172(157$ to 188$)$ & $179(160$ to 217$)$ & $0.012(-23.7$ to -3.1$)$ \\
DRvTBMD & $172)$ & \\
\hline
\end{tabular}

an increasing number of young males might benefit from the opportunity of semen cryopreservation prior to gonadotoxic therapies. However the HFEA Act (1990) deems that minors must demonstrate competence and that consent by proxy is not possible.

Aim: To audit the number of male patients offered semen cryopreservation on an adolescent cancer unit after a staff training and awareness programme and to assess their acceptance or refusal, the process of counselling and obtaining legal consent and the success or "failure" of those consenting.

Methods: Males aged 13 and over, diagnosed with malignancy between January 1999 and January 2001 were identified. The use of a teenage friendly information leaflet, documentation of pubertal assessment, measurements of baseline $\mathrm{LH}, \mathrm{FSH}$ and testosterone and assessment of Gillick competence were audited, together with refusal or acceptance of banking, the success or "failure" of those attempting, and the legal process of consent.

Results: 20 eligible patients with cancer of median age 14.5 (range 13-18) were identified, of whom 13 were offered the opportunity to bank (aged 14-18). 11/13 were offered the leaflet and 9 of these had documentation of pubertal assessment, Gillick competence and measurement of baseline hormones. 7 patients (aged 14-18) accepted and 6/7 banked successfully. 6 (aged 14-17) declined.

Summary: Despite staff training, a significant proportion (35\%) of eligible adolescents were not offered information about sperm banking. This group tended to be the youngest (= 14 years) and those most needy of expert assessment and counselling. Of those who were offered sperm banking, 15\% were not formally assessed for Gillick competence or pubertal development. Those successfully cryopreserving semen were older ( $=15$ years), but those declining were of a wide age range (14-17 years).

Conclusion: Our data confirms that adolescents can successfully bank sperm. However despite staff training, younger adolescent males (= 14 years) were unlikely to be considered for semen cryopreservation. Further training needs to emphasise that competence to bank depends on assessment of pubertal status and Gillick competence, rather than age alone.

\section{G53 DETECTION OF RELAPSE IN CHILDHOOD SOLID TUMOURS}

L.M. Howell, A. Mensah, A.M. Will, R.F. Stevens, O.B. Eden, B.M.D. Brennan, G.W.J. Makin. Department of Paediatric Oncology, Royal Manchester Children's Hospital and Medical School, University of Manchester

Aims: Frequent follow-up, often with regular investigation, is a routine part of Paediatric Oncology. Many treatment protocols specify both the type and frequency of the investigations to be performed. There is however, little evidence as to the value of such surveillance in the detection of relapse. We thus conducted a retrospective study of relapse detection in a patient cohort.

Methods: A cohort of 316 children diagnosed with solid tumours between 1992 and 1996 was identified. This patient group had a minimum follow up period of 5 years from the end of treatment. Case notes were reviewed and numbers of clinic visits and surveillance investigations recorded. For any relapse that occurred the method of relapse detection was noted.

Results: The average age of the cohort was 5.4 years, and the mean period of follow-up was 5.9 years. 25 patients were excluded because they never achieved remission, and 4 were lost to follow-up. 3417 routine clinic visits were made by this cohort. The frequency of clinic attendance varied from 26 visits per patient with Wilm's tumour, to 15 per brain tumour patient. 1672 radiological investigations were performed on this patient group over this period. $60 \%$ of these were chest radiographs, $14 \%$ were ultrasounds, and $12 \%$ were MRI scans. Wilm's tumour patients had on average 17.5 radiological investigations during this period of follow up, whilst brain tumour patients had only 5.4. 31 relapses were detected in this cohort. $61 \%$ were detected symptomatically, $26 \%$ at routine clinic visits, and $13 \%$ on routine investigation. $81 \%$ of relapses occurred $>1$ year from the end of treatment. We calculated that routine MRI scan detected 1 unsuspected relapse for each 67 scans performed. Routine CT scan detected 1 relapse for every 104 scans performed, whilst routine chest radiography detected only 1 relapse for every 334 films. The low incidence of relapse detection by routine surveillance in this cohort raises questions as to the usefulness of such follow-up in children with solid tumours. 